\title{
Palpebral oedema associated with sensitization to cobalt in a dental prosthesis
}

\author{
J. Vicente
}

\author{
A. España
}

Department of Dermatology, University Hospital, School of Medicine, University of Navarra, Pamplona, Spain

SIR, Dental prostheses can provoke adverse reactions by both immunological and nonimmunological mean, and these can result in mucous membrane and cutaneous lesions. We present a patient with a removable methacrylate dental prosthesis who had progressive oedema of the eyelids and lips. This seemed to be due to hypersensitivity to cobalt, the main metal component of the prosthesis.

A 47-year-old woman gave a 1-month history of persistent oedema of the eyelids and lips. There was no apparent relationship to externa] triggering factors, and she liad no known history of sensitization or atopy and no relevant personal or family history. For 1 year she had been wearing a partial removable methacrylate prosthesis (Fig. 1). This was mainly composed of cobalt and chromium (Co: $60 \%$, Cr: $30 \%$, Mn: $5 \%$, Si: $2 \%$. Mo: +. W: +, C: +1 . There were no subjective symptoms connected with wearing the prosthesis.

Examination showed slight oedema of the lower eyelids (Fig. 2) and the mid-upper lip. When the prosthesis was taken out, there were erythema and minimal erosions at the contact site on the hard palate (Fig. 3). Epicutaneous patch tests to the standard series of the GEIDC (Spanish Group of Contact Dermatitis), to the dental series (Chemotechnique $^{\mathrm{R}}$ ) and to the components of the prosthesis were performed. Cobalt chloride was positive on the 2nd and 4th day $(+++/+++)$, but the other substances tested were negative. Single blind oral provocation tests were performed using cobalt chloride (2.5 and $5 \mathrm{mg}$ ) and a placebo. No change was noted with the placebo, but there was slight inflammation of the eyelids with the $2.5 \mathrm{mg}$ dose of cobalt chloride, and significant oedema of the lower eyelids and upper lip with $5 \mathrm{mg}$.

When the prosthesis was removed, the patient's symptoms improved and the cutaneous and mucous membrane lesions cleared completely within 1 week. No biopsy was taken. The prosthesis was replaced by another containing no cobalt and, 6 months later, the patient had had no further oedema in the eyelids or lips.

On occasions dental prostheses can cause lesions of the skin as well as of the oral mucosa ${ }^{1}$. Urticaria ${ }^{2}$ or generalized eczema ${ }^{3}$ may occur. Recognition of these reactions is based on identifying a cause and effect relationship between the appearance of the symptoms and the insertion or changing of the prosthesis, ${ }^{4}$ positive patch tests to relevant allergens, and exclusion of other diagnoses. Cobalt is found in hard metal alloys. Isolated sensitization to cobalt, as in our patient, is unusual. Some patients with 
erosive oral lichen planus have been reported to be sensitive to cobalt, which is found in dental prostheses. ${ }^{5}$ One child, sensitive to cobalt, was reported to have developed orofacial granulomatosis. ${ }^{6}$

The mechanism by which the palpebral oedema was produced in our patient is unclear. Type IV hypersensitivity has been implicated in such reactions, ${ }^{2}$ perhaps contributed to by local factors, for example, erosions of the oral mucosa. ${ }^{6}$ Involvement of the lower eyelids and upper lip may be due to the proximity of the prosthesis and the laxity of these tissues. We suggest that the oral mucosa be examined in patients with palpebral oedema of unknown cause, and that sensitization to the components of dental prostheses should be considered in patients who wear these devices.

\section{REFERENCES}

1. Vilaplana J, Romaguera C, Cornellana F. Contact dermatitis and adversa oral mucous membrana reactions related to the use of dental prostheses. Contact Dermatitis 1994; 30: 80-4.

2. España A, Alonso ML, Soria C, Guimaraens 0, Ledo A. Chronic urticaria after implantation of two nickel-containing prostheses in a nickel allergic patient. Contact Dermatitis 1989: 21: 204-5.

3. Levantine AV, Bettley FR. Sensitivity to metal dental plate. Proc R Soc Med 1974; 67: 1007.

4. Romaguera C. Dermatitis de contacto y reacciones adversas de la mucosa oral relacionadas con el uso de prótesis dentales. Monograf Dermatol 1995; 8: 67-81.

5. Torresani C, Nannini R, Bondi A, Guadagni M, Manara GC. Erosive oral lichen planus due to sensitization to cobalt chloride. Ulin Exp Dermatol 1994; 19: 535-6.

6. Pryce DW, King CM. Orofacial granulomatosis associated with delayed hypersensitivity lo cobalt. Clin Exp Dermatol 1990: 15: 384-6. 


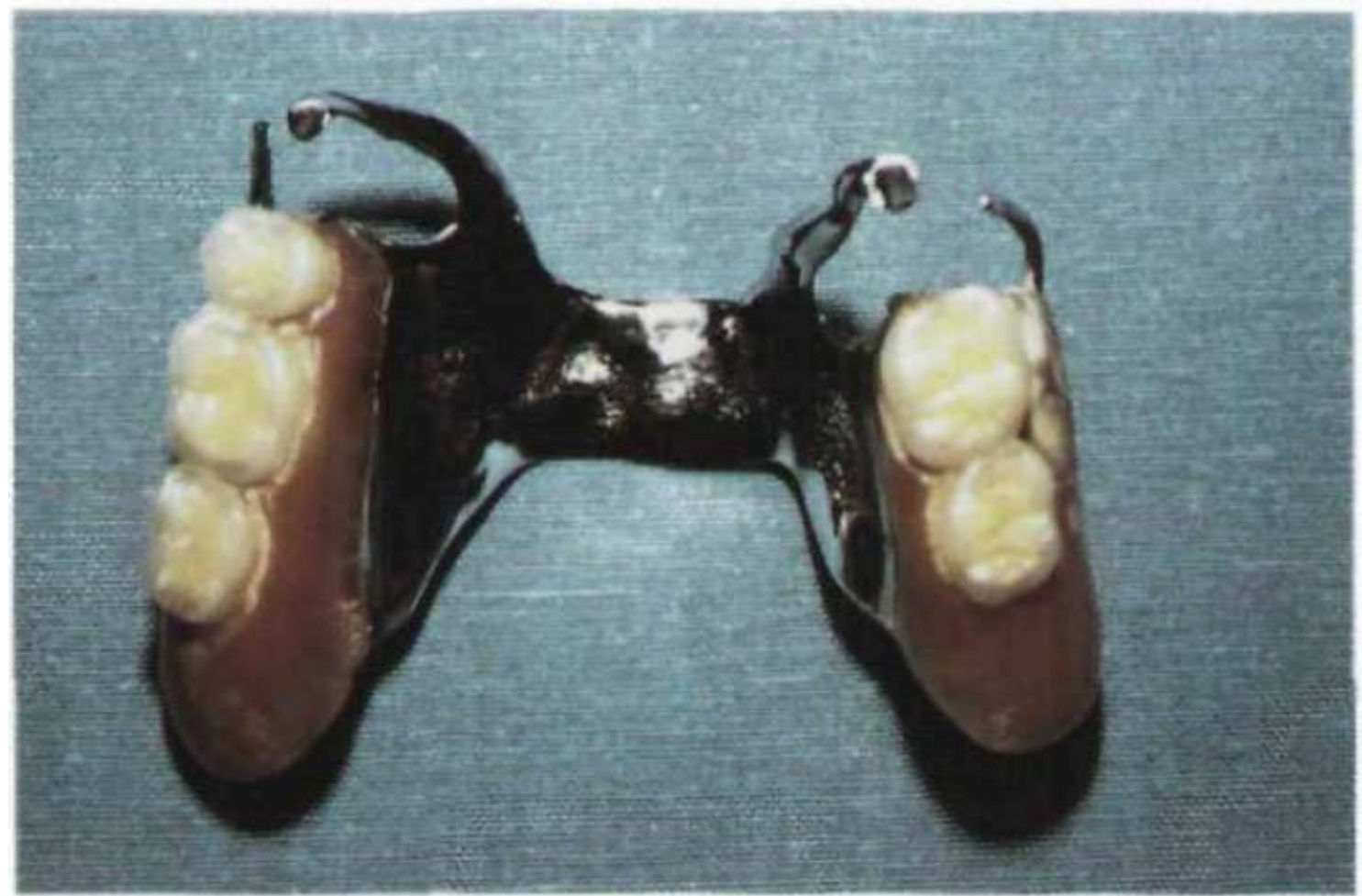

Figure 1. The partial removable dental prosthesis.

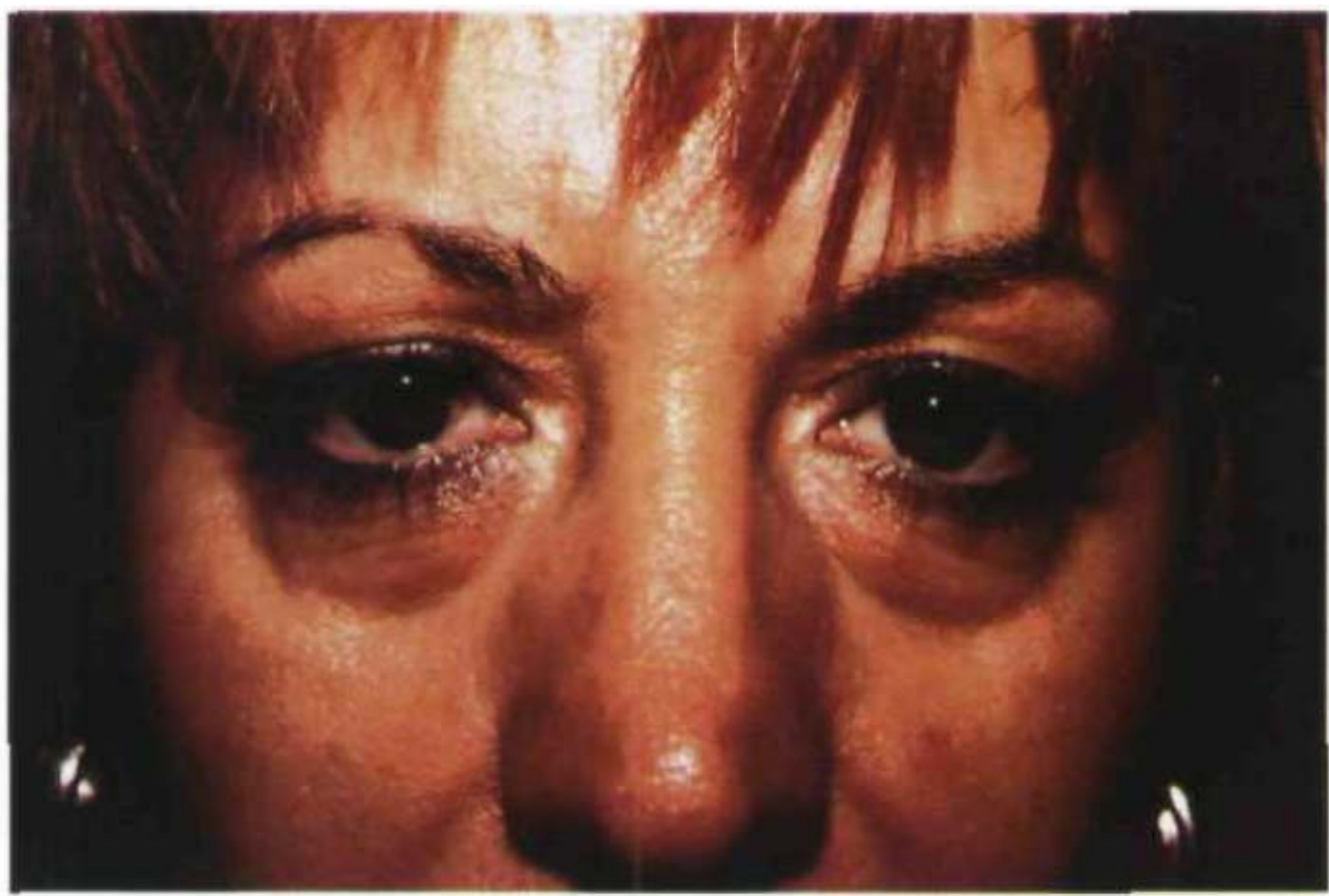

Figure 2. Slight oedema of the lower eyelids. 


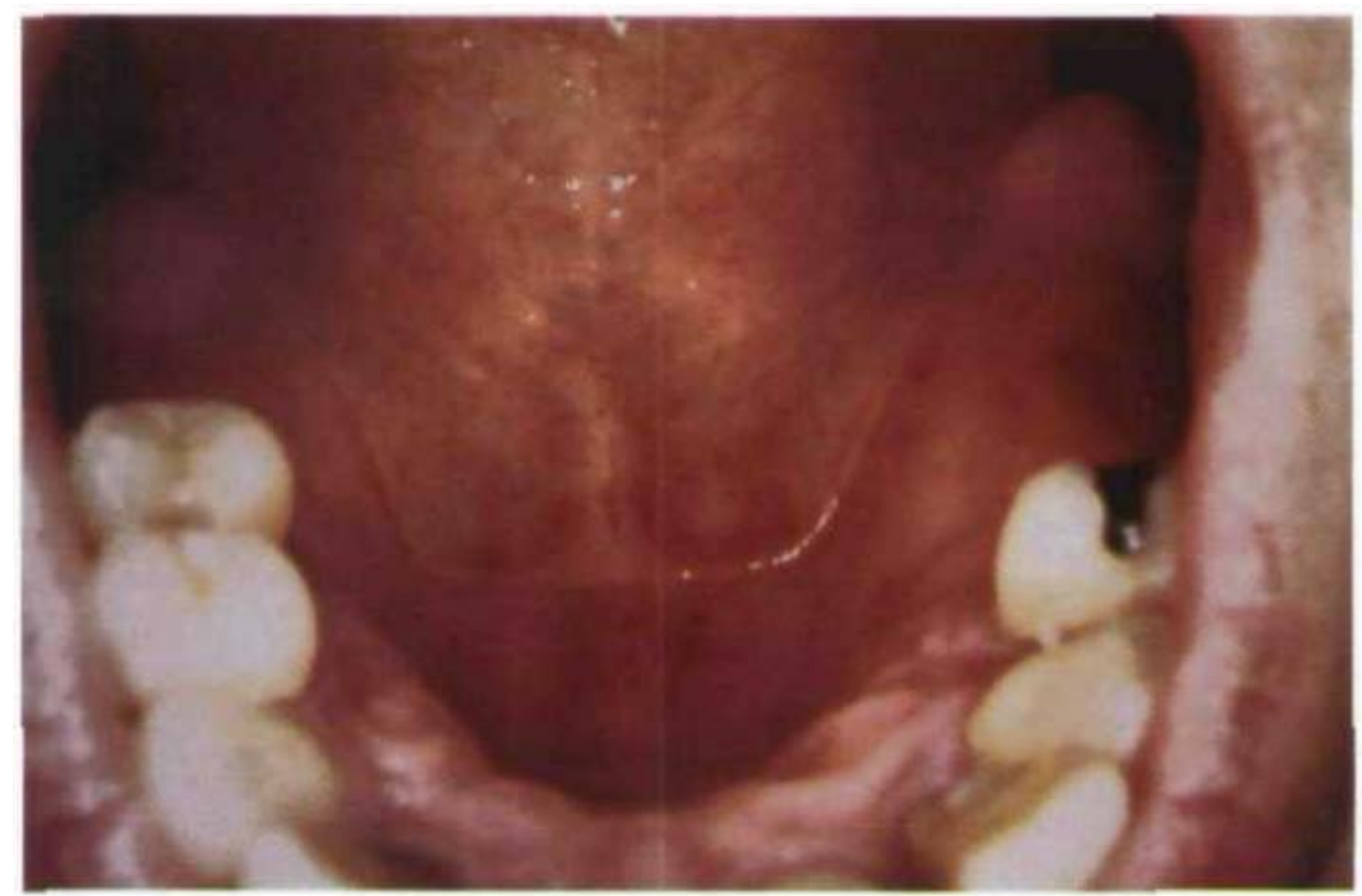

Figure 3. Minimal erosions on the hard palate, in the area in contact with the prosthesis. 